\title{
La réception du calendrier républicain dans les campagnes du Haut-Doubs. Quels enseignements ?
}

The reception of the Republican calendar in the countryside of the Haut-Doubs

\section{Danièle Pingué}

\section{(2) OpenEdition \\ 12 Journals}

\section{Édition électronique}

URL : https://journals.openedition.org/ahrf/11221

DOI : 10.4000/ahrf.11221

ISSN : 1952-403X

Éditeur :

Armand Colin, Société des études robespierristes

\section{Édition imprimée}

Date de publication : 1 septembre 2007

Pagination : 79-86

ISSN : 0003-4436

\section{Référence électronique}

Danièle Pingué, «La réception du calendrier républicain dans les campagnes du Haut-Doubs. Quels enseignements ? ", Annales historiques de la Révolution française [En ligne], 349 | juillet-septembre 2007, mis en ligne le 01 septembre 2010, consulté le 01 juillet 2021. URL : http://journals.openedition.org/ ahrf/11221 ; DOI : https://doi.org/10.4000/ahrf.11221 


\title{
LA RÉCEPTION DU CALENDRIER RÉPUBLICAIN DANS LES CAMPAGNES DU HAUT-DOUBS. QUELS ENSEIGNEMENTS?
}

Danièle PINGUÉ

\begin{abstract}
La réception du calendrier révolutionnaire dans les campagnes du district de Pontarlier (Doubs), petite région très enclavée et très attachée à la religion catholique, permet de mesurer la pénétration éventuelle de la "révolution culturelle de l'an II " dans la "France profonde " d'alors. L'étude repose sur l'exploitation de trois indicateurs : la formulation des dates dans les actes officiels locaux, le respect ou non du décadi et le choix des prénoms des nouveaux-nés. Si la nouvelle formulation des dates est adoptée avec plus ou moins de retard par les administrateurs, on constate que la population oppose une véritable résistance au décadi et ne recourt pratiquement pas aux prénoms révolutionnaires. Toutefois, cette attitude n'est perçue ni par les intéressés, ni par les autorités elles-mêmes comme une manifestation d'opposition à la Révolution dans son ensemble.
\end{abstract}

Mots-clés : calendrier révolutionnaire, décadi, prénoms révolutionnaires, Doubs (département du).

Contrairement aux affirmations de l'historiographie classique, le calendrier républicain n'a pas fait l'objet d'un rejet systématique de la part des populations rurales et a même pu connaître, en l'an II, un relatif succès. Ce fut notamment le cas dans les campagnes de l'Île-de-France, étudiées par Serge Bianchi ${ }^{1}$. Mais comment ce calendrier fut-il reçu dans des zones au profil géopolitique totalement différent ?

Notre enquête porte sur la petite région de plateaux et de montagnes située à l'Est de la Franche-Comté, adossée à la frontière suisse, que l'on appelle aujourd'hui le « Haut-Doubs » et dont la plus grande partie correspondait, sous la Révolution, au district de Pontarlier. Sur le plan géographique, à la fin du XVIII siècle, cette région était encore très enclavée :

(1) Serge Bianchi, "La bataille du calendrier ou le décadi contre le dimanche. Nouvelles approches pour la réception du calendrier républicain en milieu rural ", $A H R F, \mathrm{n}^{\circ} 312,1998$, p. 245-264; Serge Bianchi, La Révolution et la Première République au village, Paris, CTHS, 2003, p. 469-485. 
une seule route importante la reliait à l'extérieur ${ }^{2}$; à l'intérieur, la circulation était particulièrement difficile : aux contraintes toujours actuelles du relief et du climat, s'ajoutait alors le mauvais état des voies; ainsi, en hiver, certaines localités n'étaient accessibles qu'à pied. $90 \%$ de la population était rurale et la seule ville importante, Pontarlier, dépassait à peine 3200 habitants. En ce qui concerne l'esprit public, le Haut-Doubs conserve la réputation d'avoir été, en bloc, réfractaire à la Révolution. La réalité est plus complexe : comme dans l'ensemble de la Franche-Comté, la mobilisation patriotique y connut un réel succès ; il abrita un réseau non négligeable de sociétés populaircs et fournit - par exemple - l'un des meilleurs taux de participation nationale au plébiscite constitutionnel de 1793 ; ajoutons qu'en l'an II, la quasi-totalité des communes fut dotée d'un comité de surveillance. ${ }^{3}$. Rien de comparable, donc, au cas de la Vendée par exemple. Cependant, plus encore que le reste de la Franche-Comté, le Haut-Doubs se caractérisa par l'attachement inconditionnel de ses habitants à la religion catholique et opposá dès 1791 une très vive résistance à la Constitution civile du clergé. À la fin de l'été 1793, l'insurrection paysanne catholique de la "Petite Vendée ", bien qu'éphémère et circonscrite à quelques cantons, allait marquer durablement la mémoire collective.

Dans ce contexte a priori peu favorable à l'adoption du calendrier républicain, quel accueil fut réservé à son introduction, en l'an II, et comment l'interpréter? Par la suite, comment évolua son usage, de l'an III à l'an VIII ?

Pour répondre à ces questions, nous avons exploité cinq types de sources : les registres d'état civil, les délibérations municipales, les comptes décadaires des agents nationaux de l'an II et de l'an III, puis des commissaires du Directoire, les registres des comités de surveillance, et enfin, les résultats d'une enquête du district de Pontarlier en l'an II, conservés pour toutes les communes et concernant notamment l'observation du décadi. Par contre, deux sources importantes pour ce type de recherches ont manqué : les registres des sociétés populaires et les archives des justices de paix, qui n'ont malheureusement pas été conservés pour le territoire étudié.

(2) G. Arbellot, J. Bertrand, B. Lepetit, Atlas de la Révolution française, fasc. 1 : Routes et communications, Paris, EHESS, 1987, p. 15.

(3) Sources : Jean-Luc MaYAud, La Franche-Comté au XIX' siècle, Wettolsheim, Ed. Mars et Mercure, 1979 ; Jean Boutıer et Philippe Boutry (dir.), Atlas de la Révolution française, fasc. 6 : Les sociétês politiques, EHESS, Paris, 1992 ; Serge ABERDAM, L'élargissement du droit de vote entre 1792 et 1795 , thèse de doctorat sous dir. Michel Vovelle, Univ. Paris I, 2001 ; Jean-Olivier Mottet de La Fontaine, Les comités de surveillance du district de Pontarlier, mémoire de maitrise sous dir. Danièle PinguÉ, Université de Besançon, 2004. 


\section{L'accueil du nouveau calendrier en l'an II : la formulation des dates.}

Rappelons que le calendrier républicain fut adopté par la Convention le 5 octobre 1793, les noms des mois fixés le 24 octobre et enfin, son usage rendu obligatoire le 4 frimaire an II (24 novembre 1793). Au bout de combien de temps ces lois furent-elles appliquées dans le HautDoubs et après quelles hésitations?

Nous nous sommes d'abord penchée sur la nouvelle formulation des dates, en utilisant les sources classiques sur cette question : registres d'état civil et délibérations des administrations.

Le test sur les registres d'état civil a porté sur 36 communes du district de Pontarlier (1/3 du total qui était de 91), dont la population en 1790 variait de 45 habitants (Reculfoz) à 983 (Jougne) ${ }^{4}$. Nous avons relevé trois types de renseignements : la dernière date " vieux style »; la première date " républicaine ", même incorrecte ; enfin, la première date « républicaine " correctement formulée.

Dans les trois-quarts des communes, la dernière mention d'une date du calendrier grégorien est antéricure au $1^{\text {er }}$ frimaire an II (21 novembre 1793). Cette donnée doit être interprétée avec précaution, car l'intervalle qui sépare la dernière date "vieux style " de la première date « républicaine »varie, selon la démographie de la commune, de quelques jours à 7 mois ; il serait donc excessif d'affirmer que les trois-quarts des communes de notre échantillon ont adopté le calendrier républicain dès brumaire, comme c'est le cas en île-de-France. Par contre, ce dont on est certain, c'est que le $1^{\text {cr }}$ frimaire, le calendrier grégorien est encore en vigueur dans une commune sur quatre. La dernière à l'utiliser sera le petit village de Saint-Gorgon (246 habitants), avec un mariage daté de « l'an

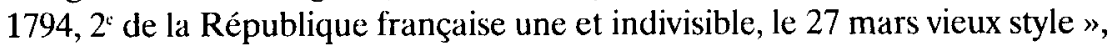
5 mois donc après le vote de la loi du 24 octobre. Fait plus surprenant, il est encore utilisé le « 18 janvier 1794 vieux style » à Jougne, chef-lieu de canton pourtant réputé pour son bon esprit public.

Si l'on considère à présent la première mention d'une date « républicaine ", on constate que le nouveau calendrier est en vigueur dans la moitié des communes à partir de nivôse (21 décembre 1793) et dans les troisquarts à partir de pluviôse (20 janvier). La première à l'adopter dans son registre d'état civil est le petit village de Bulle (416 habitants), avec une naissance datée du « $9^{c}$ jour de la première décade du second mois de l'année seconde de la République française " ( ce qui correspond au 24 octobre 1793, soit trois semaines après le premier vote de la Convention, le jour même où celle-ci adopte les noms des mois).

(4) AD Doubs, 5 Mi R 734, 735, 738, 766, 768, 769, 774, 782, 783, 784, 785, 786, 790, 814, 815, 816. 
Un peu plus de la moitié des officiers ou des greffiers (20 sur 36 ) adoptent directement la bonne formulation des nouvelles dates (si l'on excepte l'orthographe) ; les autres se livrent à des tâtonnements plus ou moins longs (qui vont de deux semaines à un an). Les « erreurs » sont les suivantes : rajout du millésime du calendrier grégorien : 7 cas ; ignorance du nom du mois : 4 cas ; rajout de la concordance complète avec le calendrier grégorien : 3 cas; ratures : 1 cas ; utilisation de l'un ou l'autre calendrier selon le rédacteur de service : 1 cas (Courvières).

Au total, malgré les imprécisions liées au caractère aléatoire des données, il est indéniable que dans le Haut-Doubs, l'introduction du calendrier républicain dans les actes d'état civil connaît un certain retard par rapport à ce que l'on peut constater, par exemple, en Île-de-France; tandis que les communes étudiées par Serge Bianchi l'utilisent massivement, d'une manière correcte, à partir de nivôse an II (21 décembre 1793), ici, à cette date, à peine la moitié des localités sont dans ce cas. Toutes, cependant, finissent par l'adopter.

Plus qu'une manifestation de résistance à la Révolution, cette situation peut s'expliquer par les difficultés des communications soulignées en introduction et le temps que mettent les lois à parvenir dans les communes. Les registres des municipalités et des comités de surveillance sont à ce sujet éclairants : si l'application du nouveau calendrier y est plus rapide que dans les registres d'état civil (dès brumaire dans la majorité des cas), on note des différences selon les lieux, dues d'une manière évidente au délai de réception des lois ; ainsi, à Chaffois (canton de Pontarlier, 625 habitants), le décret du « $16^{\circ}$ jour du premier mois relatif aux dates des actes publics» (celui du 5 octobre 1793, avec d'ailleurs une légère erreur de concordance) est publié seulement au bout d'un mois, le 10 novembre, et mis en application pour dater les réunions encore quinze jours plus tard, le 4 frimaire an II (24 novembre) $)^{5}$. De même, le décret du 4 frimaire an II (24 novembre 1793) qui refond en un seul les différents textes de loi sur le calendrier, n'est publié que le 20 ventôse (10 mars 1794 - trois mois et demi plus tard) dans les petites communes de Ouhans (canton de Goux, 556 habitants) et de Oye et Pallet (canton de Pontarlier, 245 habitants), où il est d'ailleurs remis à l'instituteur'.

Le manque d'instruction de certains administrateurs intervient également et permet d'expliquer notamment le seul cas de retour en arrière que nous avons rencontré : dans le petit village de Reculfoz (canton de Mouthe, 45 habitants), le greffier, après avoir laborieusement daté un mariage du «vainte un prerestalle l'an secon de la République française

(5) AD Doubs, EAC, $1150 \mathrm{Dl}$.

(6) AD Doubs, L 1282. 
une et indivisible ", puis un décès de "l'an 1795, l'an III de la République

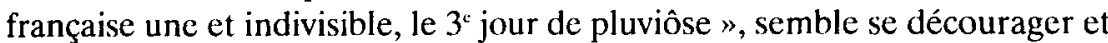
revient, en "l'an IV de la République " au " 2 septembre vieux style " puis au « 22 décembre vieux style »; il faut attendre l'an V, et l'apparition des registres imprimés, pour que la formulation redevienne correcte.

Ce test sur la formulation des dates ne nous renseigne, naturellement, que sur l'attitude des fonctionnaires ou des administrateurs et il n'y a rien d'étonnant à ce qu'ils finissent tous par appliquer la loi, puisque tel est précisément leur rôle. Mais quelle est l'attitude de l'ensemble de la population?

Pour tenter de l'apprécier, deux autres indices ont été mis à contribution : l'accueil du décadi et le choix des prénoms.

\section{Le décadi et les prénoms}

Une enquête du district de Pontarlier du 21 floréal an II (10 mai 1794), dont les résultats ont été conservés pour les 91 communes, pose notamment la question suivante : «Faites-vous publier les lois chaque jour de décade ? Dans quel endroit ? Les habitants de votre commune emploient-ils ce jour-là à adorer l'Être suprême et au repos? »?

Les réponses des communes sont édifiantes : un peu plus de la moitié des municipalités affirment qu'elles respectent le décadi pour la publication des lois ; mais $1 / 3$ disent les publier au fur et à mesure ; quelques-unes les publient à la fois le dimanche et le décadi ; enfin, près d'une sur dix ne les publient que le dimanche, et tiennent à l'expliquer : à La Grande Combe et à Lac ou Villers (aujourd'hui Villers le Lac, canton de Morteau) : « On faisait publier les lois les jours de décade, personne ne venait, on a quitté "; dans trois communes du canton de Mouthe, on a choisi « le dimanche pour leur donner plus de publicité ». Les municipalités des chefs-lieux de canton font pourtant des efforts en organisant ce jour-là des « cours de morale » et ceux de Mouthe, notamment, attirent des citoyens de plusieurs communes environnantes ${ }^{8}$. Cependant, pour la grande majorité des citoyens, le décadi est un jour comme un autre.

En effet, la résistance des habitants au repos décadaire est particulièrement forte : moins d'une commune sur cinq affirme le respecter et près des trois-quarts, par contre, répondent non à la question. Le plus intéressant est que cette situation ne choque absolument pas les autorités. À une exception près, aucune plainte à ce sujet n'est formulée par les comités de surveillance, pourtant actifs par ailleurs. Et l'exception qui confirme la

(7) AD Doubs, L 1279

(8) Le Crouzet, Métabief - bien que dans le canton de Jougne -, Petite-Chaux , Les Pontets, Rondefontaine, Sarrageois ; de méme, des citoyens de Rochejean se rendent à Labergement. 
règle est pour le moins ambiguë : la citoyenne Françoise Droz, du village de Boujailles, se voit reprocher d'avoir travaillé à son métier de " tissière " au mépris de la loi, le 20 nivôse, jour de décade, fête annoncée par « la loi en action de grâce de la prise de Port la Montagne, ci-devant Toulon "9. La question ne semble pas préoccuper non plus les représentants en mission qui, à notre connaissance, ne l'évoquent jamais ${ }^{10}$.

Le test des prénoms est encore plus révélateur de l'attitude des populations. Il a été effectué à partir des registres d'état civil mentionnés plus haut ; nous avons retenu la distinction proposée par Michel Vovelle et Claude Langlois entre les prénoms de rupture, les prénoms de compromis (prénom double, dont chaque partic appartient à un calendrier différent) et les prénoms ambigus (appartenant aux deux calendriers)". Sur les 666 enfants nés dans les 36 communes concernées entre $1^{\text {cr }}$ janvier 1793 et la fin de l'an II (21 septembre 1794) aucun n'a reçu un prénom de rupture et les quatre prénoms de compromis $(0,6 \%)$ qui ont été relevés sont plutôt l'exception qui confirme la règle ${ }^{12}$. Certes, s'y ajoutent quarante et un prénoms ambigus (6\%) : mais Rose, Victoire et Victor étaient déjà présents auparavant, et une étude plus précise serait nécessairc pour savoir si leur fréquence a augmenté.

Pour résumer, si l'introduction du calendrier révolutionnaire dans les campagnes du Haut-Doubs ne soulève aucune difficulté particulière à propos de la datation des actes officiels, elle entraîne une véritable résistance de la population, avec la complicité des autorités constituées, en ce qui concerne la tentative de remplacer le dimanche par le décadi, et n'a pratiquement aucun impact sur le choix des prénoms des nouveaux nés.

Cette résistance va se confirmer et s'amplifier de l'an III à l'an VIII.

\section{L'évolution de l'an III à l'an VIII.}

Des communes qui avaient tenté en l'an II de "solemniser " le décadi, y renoncent assez vite en l'an III, avec parfois des attitudes plutôt

(9) AD Doubs, L 1280.

(10) Gilles ARCHIMBAUD. Les représentants en mission dans le département du Doubs (1793 - an IV). mémoire de maîtrise sous dir. Michel BIARD, Univ. Paris 1, 2000 ; Emmanuel Grosperrin, La mission du représentant Lejeune dans les déparements du Doubs, du Jura et de la Haute-Saône en l'an II, mémoire de maîtrise sous dir. Danièle Pingué, Univ. Besançon, 2002.

(11) Claude LangloIS, Timothy TACKETT, Michel Vovelle et Alii, Atlas de la Révolution française, fasc. 9, Religion, Paris, EHESS, 1996, p. 46. Un état complet de la question est présenté dans les $A H R F, \mathbf{n}^{\circ}$ 322, 2000, avec des articles de Alain Crolx, Dominique Julia, Serge Bianchi, Pierre-Henty Bicly, Raphael Bange, Jean-Pierre Lethutluter, Philippe Daumas.

(12) À Jougne, Marie-Joseph Égalité Burlet, fille d'un journalier, le 1" mai 1793 ; à Aubonne, Rose-Liberté Pourchet, fille d'un cultivateur, le 3 ventôse an II et et Marie-Joseph Égalité Grandguillaume, fille d'un journalier, le 9 ventôse an II ; enfin, à Gilley, le I0 novembre 1793, Dcnis Joseph Aimable Alexis Fortunet François Marat Lepeletier, fils de Denis Alexis Cattet, laboureur, commandant du bataillon nord du canton de Montbenoît. 
surprenantes de la part des autorités constituées. Ainsi, à Aubonne, dont la municipalité a pourtant fait appliquer consciencieusement les lois en l'an II, dès le 23 thermidor an II (qui correspond au dimanche 10 août), le maire annonce que les séances du conseil général auront lieu désormais le dimanche ; à partir du 14 floréal an III (dimanche 3 mai 1795), ce jour remplace le décadi pour la publication solennelle des lois ; enfin, en thermidor an III, la même municipalité décide de célébrer avec une journée d'avance la commémoration de la journée du 10 Août, afin qu'elle tombe un dimanche ${ }^{13}$.

Dans leurs comptes décadaires, conservés d'une manière exhaustive pour les neuf cantons du district de Pontarlier, les commissaires du Directoire vont revenir régulièrement sur le sujet en précisant et en éclairant a posteriori l'attitude de la population et des autorités en l'an $\mathrm{II}^{14}$.

En ce qui concerne le calendrier proprement dit, celui-ci est respecté dans les actes publics ainsi que dans les actes sous seing privé, par crainte de leur nullité dans le cas contraire. Mais il n'est pas utilisé dans les conversations privées, où, selon l'expression d'un commissaire, le peuple « compte toujours les jours et les mois de l'ancien calendrier ${ }^{15}$. Pour les observateurs, ce phénomène doit toutefois être dissocié du "fanatisme ", et s'expliquerait plus simplement par le fait que « les gens de la campagne ne peuvent pas s'y habituer ${ }^{16}$.

Il n'en va pas de même pour la résistance généralisée au décadi attribuée, elle, à l'action des prêtres. Dès l'an IV, tous les commissaires signalent que l'on travaille les jours de décade, alors que l'on chôme le dimanche. Les fêtes décadaires sont très peu fréquentées. Quelques "progrès » semblent se dessiner en l'an VII, liés à la crainte de la répression, essentiellement des amendes, qui frappe désormais les contrevenants ; on continue, certes, à travailler le décadi, mais en se cachant.

Cela dit, les commissaires qui dénoncent ces faits n'y voient pas une source réelle de danger pour la République. Tous constatent que l'esprit public est " bon et tranquille » et que les lois sont respectées, à l'exception, disent-ils, de «celles qui sont contraires au fanatisme " ${ }^{17}$. Ce «fanatisme » est pour la plupart des habitants de la région " comme une seconde nature ; quelque effort qu'on fasse, il n'est guère possible de le détruire ». Mais, ajoute le commissaire de Frasne, si « la manière de penser des gens de ce canton ne doit pas être envisagée comme bien convenable, [...] elle ne doit

(13) AD Doubs, EAC, 4952 D1.

(14) AD Doubs, L 224 - L 230.

(15) AD Doubs, L 224, Canton de Frasne, thermidor an VI.

(16) lbid., floréal an VII.

(17) Ibid., messidor an VI ; les autres commissaires (à l'exception de celui de Mouthe) font les mêmes constatations, mais les expriment moins clairement. 
pas non plus être regardée comme bien mauvaise. On ne rétrograde pas dans le sentier de la Révolution, mais on y marche à pas lents et bien mesurés ${ }^{18}$. Pour celui de Morteau (canton qui semble détenir le record de la résistance catholique), "malgré tout, autant que je connais les fanatiques de ce canton, je suis persuadé qu'ils ne commenceraient jamais les premiers à donner l'exemple de l'insurrection ${ }^{14}$.

Comme on pouvait s'y attendre, l'exploration des registres d'état civil de l'an III à l'an VIII dans notre échantillon de 39 communes n'a permis de découvrir aucun nouveau prénom de rupture. Des prénoms ambigus sont toujours présents en petit nombre, avec les problèmes d'interprétation rappelés plus haut; enfin, il serait intéressant d'approfondir la recherche sur les quelques prénoms ambigus " contre-révolutionnaires " tels que Reine, Reine-Antoinette ou encore Louise-Antoinette-Adélaïde (Frasne, messidor an VI).

Ainsi, le Haut-Doubs offre l'exemple d'une petite région où, conformément à la vision traditionnelle, l'introduction du calendrier républicain se heurte effectivement à la résistance voire au refus des populations rurales. Mais ce refus n'est perçu ni par les intéressés, ni par les autorités ellesmêmes comme une manifestation d'opposition à la Révolution dans son ensemble. Tel est le principal enseignement de cette brève étude qui mériterait d'être approfondie pour tenter d'appréhender dans toute leur complexité les manifestations de l'hypothétique " révolution culturelle de l'an II » dans la France rurale «profonde ».

Danièle PINGuE

Laboratoire des Sciences historiques, Université de Besançon 32 rue Mégevand -25000 Besançon daniele.pingue@wanadoo.fr 